\title{
RadioAstron orbit determination and evaluation of its results using correlation of space-VLBI observations
}

M. V. Zakhvatkin a,*, A. S. Andrianov ${ }^{\mathrm{b}}$, V. Yu. Avdeev ${ }^{\mathrm{b}}$, V. I. Kostenko ${ }^{\mathrm{b}}$, Y. Y. Kovalev ${ }^{\mathrm{b}}$, S. F. Likhachev ${ }^{\mathrm{b}}$, I. D. Litovchenko ${ }^{\mathrm{b}}$, D. A. Litvinov ${ }^{\mathrm{c}, \mathrm{b}, \mathrm{e}}$, A. G. Rudnitskiy ${ }^{\mathrm{b}}$, M. A. Shchurov ${ }^{\mathrm{b}}$, K. V. Sokolovsky ${ }^{\mathrm{b}}$, V. A. Stepanyants ${ }^{\mathrm{a}}$, A. G. Tuchin ${ }^{\mathrm{a}}$, P. A. Voitsik ${ }^{\mathrm{b}}$, G. S. Zaslavskiy ${ }^{\mathrm{a}}$, V. E. Zharov ${ }^{\mathrm{c}, \mathrm{d}}$, V. A. Zuga ${ }^{\mathrm{b}}$

\author{
${ }^{a}$ Keldysh Institute of Applied Mathematics RAS, Miusskaya sq. 4, Moscow, Russia 125047 \\ ${ }^{b}$ Astro Space Center of Lebedev Physical Institute of RAS, 84/32 Profsoyuznaya st., Moscow, Russia 117997 \\ ${ }^{c}$ Sternberg Astronomical Institute, Lomonosov Moscow State University, Universitetsky pr. 13, Moscow, Russia 119991 \\ ${ }^{d}$ Faculty of Physics, Lomonosov Moscow State University, Leninskie Gory, Moscow, Russia 119991 \\ ${ }^{e}$ Bauman Moscow State Technical University, 2-ya Baumanskaya 5, Moscow, Russia 105005
}

\section{Abstract}

A crucial part of a space mission for very-long baseline interferometery (VLBI), which is the technique capable of providing the highest resolution images in astronomy, is orbit determination of the missions space radio telescope(s). In order to successfully detect interference fringes that result from correlation of the signals recorded by a ground-based and a space-borne radio telescope, the propagation delays experienced in the near-Earth space by radio waves emitted by the source and the relativity effects on each telescopes clock need to be evaluated, which requires accurate knowledge of position and velocity of the space radio telescope. In this paper we describe our approach to orbit determination (OD) of the RadioAstron spacecraft of the RadioAstron space-VLBI mission. Determining RadioAstrons orbit is complicated due to several factors: strong solar radiation pressure, a highly eccentric orbit, and frequent orbit perturbations caused by the attitude control system. We show that in order to maintain the OD accuracy required for processing space-VLBI observations at cm-wavelengths it is required to take into account the additional data on thruster firings, reaction wheel rotation rates, and attitude of the spacecraft.We also investigate into using the unique orbit data available only for a space-VLBI spacecraft, i.e. the residual delays and delay rates that result from VLBI data processing, as a means to evaluate the achieved OD accuracy. We present the results of the first experience of OD accuracy evaluation of this kind, using more than 5,000 residual values obtained as a result of space-VLBI observations performed over 7 years of the RadioAstron mission operations.

Keywords: RadioAstron, orbit determination, space-VLBI

\section{Introduction}

The RadioAstron spacecraft, equipped with a $10-\mathrm{m}$ space radio telescope (SRT), was launched into a highly elliptical Earth orbit in July 2011 by means of a Zenit-3F rocket and Fregat-SB upper stage. The main purpose of the spacecraft is to conduct observations of galactic and extragalactic radio sources in conjunction with ground-based radio telescopes forming a multi-antenna ground-space radio interferometer with extremely long baselines Kardashev et al. (2013). The spacecraft also made it possible to test the Einstein Equivalence Principle thanks to its eccentric orbit and a highly stable on-board hydrogen maser frequency standard Litvinov et al. (2018); Nunes et al. (2019).

The SRT observes at the four frequency bands: P-, L-, C- and K. During observations the RadioAstron spacecraft

*Corresponding author at: Keldysh Institute of Applied Mathematics, Russian Academy of Sciences, Miusskaya sq. 4, 125047 Moscow, Russia.

Email address: zakhvatkin@kiam1.rssi.ru (M. V. Zakhvatkin) transmits the science data being collected in real time to a tracking station on Earth using its 1.5-m high-gain dish antenna via a $15 \mathrm{GHz}$ downlink. The science payload of the SRT includes two hydrogen maser frequency standards (H-masers) with only one of the two allowed to operate at a time. Shortly after launch one of the two H-masers was identified as impaired, while the other fully functional. The latter $\mathrm{H}$-maser was thus used since the beginning of the mission operations in 2011 till August 2017, when it exhausted its hydrogen supply and was switched off, thereby exceeding its expected lifetime by a factor of two. The science payload of the SRT and its radio downlinks may be synchronized either to the on-board H-maser signal or to a ground H-maser signal uplinked from one of the RadioAstron mission's tracking stations. While the on-board H-maser was operational, its signal, being slightly more stable than that of the uplink, was used as a reference both for the science payload and the downlink signals, including those analyzed in this paper.

A C-band radio link is used for telemetry and command 
as well as to obtain range and Doppler tracking data for the orbit determination needs. Routine tracking is performed every $2-3$ days by the two antennas located in Russia: the 64-m antenna at Bear Lakes (near Moscow) and the 70-m antenna at Ussuriysk (Primorsky Krai). RadioAstron is also equipped with a retroreflector array, which makes it possible to perform satellite laser ranging on it.

RadioAstron's orbit is highly elliptic, with the geocentric distance varying from 7,000 to $351,600 \mathrm{~km}$ and an average period of 8.6 day. The orbit of the spacecraft significantly evolves with time due to the gravitational pull of third bodies. The orbital parameters change periodically with a period of about 2 years, which provides for rich opportunities of observing various radio sources distributed all over the sky.

VLBI data processing involves the so-called fringe search procedure, i.e. the search for a maximum of the crosscorrelation of the signals recorded at different telescopes. This procedure requires the delay in arrival times of the wavefront at each pair of telescopes to be accurately known for the duration of the observation. The initial guess for the delay is calculated according to a model which utilizes various kinds of data: trajectories of the phase centers of the participating telescopes in an inertial reference frame, offsets and drifts of their clocks, propagation media parameters, etc. In order to be able to perform the fringe search in a reasonable amount of time, e.g. tens of minutes, using a modern computing cluster, a priori uncertainty in the parameters of the model should be relatively small and known (the required level of uncertainties depends on many factors, a few examples are given below).

In space-VLBI the major uncertainty in the modeled delay and its derivatives comes from orbit reconstruction errors of the spacecraft(s) carrying the radio telescope(s). For the RadioAstron spacecraft the following requirements to the OD accuracy were established prior the launch:

- position error less than $600 \mathrm{~m}$;

- velocity error less than $2 \mathrm{~cm} / \mathrm{s}$;

- acceleration error less than $10^{-8} \mathrm{~m} / \mathrm{s}^{2}$.

These requirements are determined by several factors, including the following: the wavelength of the observation, the data bit rate, the number of participating telescopes, the correlator design, the available computational resources, etc.

The requirement on the position accuracy formally reflects the delay search window with 128 spectral channels. The actual window size and the number of channels used by the correlator is significantly larger, e.g. 2048 spectral channels for the survey of active galactic nuclei (AGN), which allows to account for different kinds of errors. The velocity error less than $2 \mathrm{~cm} / \mathrm{s}$ allows to perform a fringe search on the smallest wavelength $(1.35 \mathrm{~cm})$ using the integration time of only $1 / 8 \mathrm{~s}$ in the correlator. Rather stringent requirement on the acceleration accuracy initially aroused from the necessity of keeping the phase error of a K-band signal over integration interval of $\sim 10$ minutes within a fraction of a radian. However the acceleration error within $\pm 1.5 \times 10^{-6} \mathrm{~m} / \mathrm{s}^{2}$ window can be compensated by the correlator during the processing.

For an earlier, and so far the only other, realized spaceVLBI mission of VSOP/HALCA the OD requirements were similar (1- $\sigma$ errors are given): position - $80 \mathrm{~m}$, velocity $0.43 \mathrm{~cm} / \mathrm{s}$, acceleration $-6 \times 10^{-8} \mathrm{~m} / \mathrm{s}^{2}$ You et al. (1998). The similarity is easily understandable as VSOP/HALCA was designed to observe at comparable wavelengths, 1.35, 6 and $18 \mathrm{~cm}$, and recorded the data at the same bit rate as RadioAstron (128 Mbps). Despite the fact that scientific observations with HALCA were routinely taken only at 1.6 and $5 \mathrm{GHz}$ because of sensitivity problems at 22 $\mathrm{GHz}$, its OD requirements were formulated for all three bands. Slightly more stringent requirements of VSOP to position and velocity determination accuracies are due to a different correlator design.

Orbit determination of the RadioAstron spacecraft is complicated by limited tracking support and significant non-gravitational perturbations caused by solar radiation pressure (SRP) and autonomous firings of thrusters of the spacecraft attitude control system. A unique feature of a space-VLBI spacecraft is the possibility of verifying its OD accuracy by using the so-called residual delays and delay rates obtained as a result of successful detections of interference fringes and post-correlation analysis. In this work we summarize the first experience of OD accuracy evaluation based on using residual data of space-VLBI observations. Contrary to the line-of-sight tracking observations this kind of data allows to measure errors of the spacecraft position and velocity projected on different directions. This analysis allowed us to quantitatively evaluate the accuracy of the two versions of the OD algorithm used.

\section{Orbit and dynamics}

The RadioAstron spacecraft was launched into a highly elliptic orbit with apogee distance varying between 280000 and $351000 \mathrm{~km}$, orbital period between 8.1 and 10.2 days and perigee height above $650 \mathrm{~km}$. The orbit evolves significantly with time in both eccentricity and orientation of the orbital plane because of luni-solar gravitational perturbations and due to the influence of Earth's gravitational field during occasional low perigees (Table 1). An important aspect of such orbit for space-VLBI is that it provides for observing a large sample of galactic and extragalactic radio sources at a wide range of projected baselines. Only three trajectory correction maneuvers have been performed so far. Their goal was to prolong the lifetime of the orbit by preventing reentry while passing an upcoming local minimum of the perigee height and also to prevent unacceptably long shadowing of the spacecraft.

Except for the rare moments when the spacecraft is moving near a perigee and the perigee height is near its 
Table 1: Evolution of the orbital elements of the RadioAstron spacecraft.

\begin{tabular}{|c|c|c|c|c|c|c|c|}
\hline Epoch & $2011 / 08 / 21$ & $2012 / 10 / 13$ & $2014 / 01 / 27$ & $2015 / 03 / 23$ & $2016 / 06 / 24$ & $2017 / 07 / 31$ & $2018 / 02 / 17$ \\
\hline Perigee height, $10^{3} \mathrm{~km}$ & 6.213 & 69.265 & 1.075 & 67.666 & 0.654 & 75.716 & 3.699 \\
\hline Apogee height, $10^{3} \mathrm{~km}$ & 336.526 & 278.126 & 338.611 & 280.593 & 336.278 & 306.435 & 329.026 \\
\hline$i, \operatorname{deg}$ & 56.354 & 76.804 & 9.199 & 56.692 & 43.866 & 68.527 & 51.309 \\
\hline$\Omega$, deg & 331.682 & 295.970 & 152.397 & 107.007 & 0.930 & 306.140 & 297.661 \\
\hline
\end{tabular}

local minimum, the major perturbing accelerations are due to gravitational pull of third bodies and non-gravitational accelerations. The latter are the main source of errors when modeling the dynamics of the RadioAstron spacecraft. Of the non-gravitational perturbations SRP has the greatest impact on the orbit.

Because of its 10-m on-board antenna, the area-tomass ratio of the RadioAstron spacecraft is as high as 0.03 $\mathrm{kg} / \mathrm{m}^{2}$. Depending on the spacecraft orientation with respect to the Sun this results in accelerations due to SRP as high as $2 \cdot 10^{-7} \mathrm{~m} / \mathrm{s}^{2}$. Accounting for SRP is additionally complicated by the fact that the spacecraft has to change its attitude according to the direction to the radio source to be observed, thus the SRP may vary significantly over a time span of several hours.

In addition to direct acceleration, SRP produces the major part of the perturbing torque. Attitude control of the RadioAstron spacecraft, which includes compensation of external torques, is implemented using reaction wheels. The spacecraft attitude in the inertial reference frame can be roughly described as piece-wise constant - most of the time the spacecraft is not rotating, with external torques compensated by the rotation of the reaction wheels, the changes of attitude occur relatively quickly, with their duration of order of a few minutes.

Several times a day the reaction wheels are desaturated. This happens either due to the angular momentum accumulated by the reaction wheels reaching its maximum allowed value, so that they cannot parry the external torque any further, or due to the need to perform a significant attitude change which would not be possible using the reaction wheels alone in their current state. Desaturating the reaction wheels implies stopping them almost completely with resulting rotation of the spacecraft prevented by means of thrusters. Every desaturation produces a net Delta $\mathrm{V}$ of $3-7 \mathrm{~mm} / \mathrm{s}$. Total effect of the desaturations on the orbit is comparable to the one of direct SRP and therefore must be taken into account.

In order to properly take the SRP influence into account, we have developed an adjustable model, which allows us to calculate both the perturbing acceleration and torque for a given attitude with respect to the Sun Zakhvatkin et al. (2014). The SRP model utilizes a simplified model of the spacecraft surface, which consists of the 10-m parabolic dish antenna, the solar panels and the spacecraft bus (Fig. 1). The coefficients of reflectivity $\alpha_{1}$ and specularity $\mu_{1}$ are associated with sunlit surfaces of the antenna and spacecraft bus, the coefficient of reflectivity $\alpha_{2}$ - with the surface of the solar panels. According to the model, the force and torque due to SRP in the spacecraft-fixed frame are

$$
\begin{aligned}
\mathbf{F}_{S R P} & =\alpha_{1}\left(1-\mu_{1}\right) \mathbf{F}_{A, B}^{d}(\mathbf{s})+\alpha_{1} \mu_{1} \mathbf{F}_{A, B}^{s}(\mathbf{s}) \\
& +\left(1-\alpha_{1}\right) \mathbf{F}_{A, B}^{a}(\mathbf{s}) \\
& +\alpha_{2} \mathbf{F}_{S P}^{s}(\mathbf{s})+\left(1-\alpha_{2}\right) \mathbf{F}_{S P}^{a}(\mathbf{s}) \\
\mathbf{M}_{S R P} & =\alpha_{1}\left(1-\mu_{1}\right) \mathbf{M}_{A, B}^{d}(\mathbf{s})+\alpha_{1} \mu_{1} \mathbf{M}_{A, B}^{s}(\mathbf{s}) \\
& +\left(1-\alpha_{1}\right) \mathbf{M}_{A, B}^{a}(\mathbf{s}) \\
& +\alpha_{2} \mathbf{M}_{S P}^{s}(\mathbf{s})+\left(1-\alpha_{2}\right) \mathbf{M}_{S P}^{a}(\mathbf{s})
\end{aligned}
$$

Here, $\mathbf{s}$ is the Sun vector, $\mathbf{F}()$ and $\mathbf{M}()$ on the right-hand side of Equations (1) and (2) represent the contributions to, respectively, the force and torque from various parts of the spacecraft, which we denoted by the following subscripts: $A, B$ - the antenna and the spacecraft bus, $S P$ solar panels. The superscripts $d, s$ and $a$ denote, respectively, diffuse reflection, specular reflection and absorption of the solar radiation.

The surface of the spacecraft bus and the solar panels in the model consists of a number of rectangular elements. Provided that all restrictions on the spacecraft attitude are met, these elements do not shadow each other from the Sun, which facilitates the calculation of the force and torque components. Surface of the parabolic antenna of the SRT is represented with 4096 flat triangular elements. Each element of the antenna surface is considered sunlit if other parts of the antenna or rectangular elements of the spacecraft bus or solar panels do not shadow the geometric center of the element. Thus the surface of the spacecraft in the model consists of flat elements, for each of which the SRP force due to absorption, specular reflection and diffuse reflection of radiation is calculated using the area of the element, its normal vector and unit vector towards the Sun. Summation over all sunlit elements provides $\mathbf{F}()$ and $\mathbf{M}()$ components in the right-hand sides of Equations (1) and (2). These components depend only on the position of the Sun, s, therefore, they are tabulated for various Sun angles to speed up the integration of equations of motion.

Although this model describes the direct SRP perturbation, we believe that adjustable coefficients allow it to also account for the major part of the force due to thermal radiation from the spacecraft surface.

We do not use the torque estimation provided by Equation (2) in the model of motion of the spacecraft's center of mass. However, unlike the SRP force the torque, as will be shown below, can be measured directly. Since the calculated value of the torque depends on the SRP coefficients, observations of the torque can be used in the 


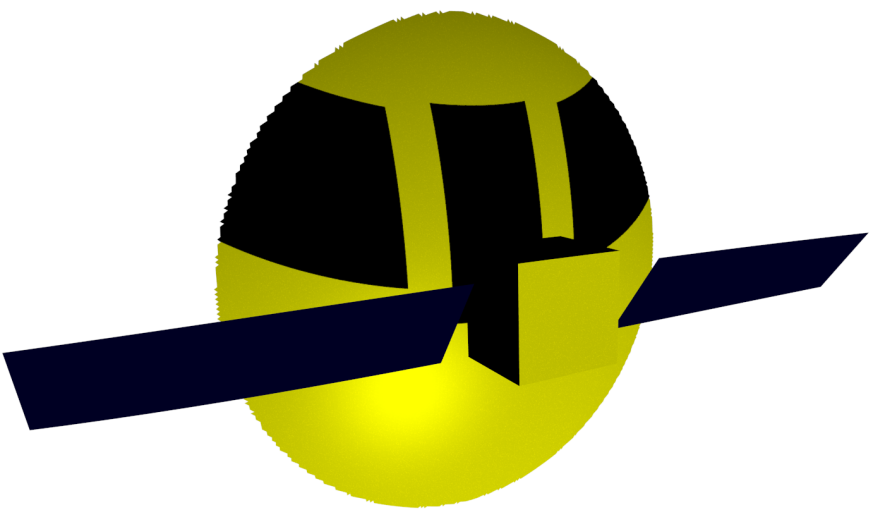

Figure 1: The approximation of the RadioAstron spacecraft surface used in the SRP model

OD to improve the estimate of the SRP coefficients. The SRP coefficients are assumed constant during the whole orbit determination interval, which usually spans for 2030 days.

The complete specification of the dynamic model we use to describe the orbital motion of the RadioAstron spacecraft is given in Table 2. Between the events of reaction wheel desaturation (momentum dumps) the spacecraft moves passively and every desaturation event is associated with a certain Delta $\mathrm{V}$ vector. A priori values of velocity change vectors are calculated using the attitude data available via telemetry and the target values of Delta $\mathrm{V}$ reported by the attitude control system for each thruster firing event. The model for the atmospheric drag is significantly simpler than the SRP model, because most of the time it has close to zero impact on the motion. For the whole duration of the mission the perigee height was below $1500 \mathrm{~km}$ (the nominal height of the atmosphere in the density model used) only several times.

\section{Tracking and on-board observations}

Several types of observational and telemetry data are used to determine RadioAstron's orbit. Standard radio tracking consists of range and Doppler measurements at the C-band. This tracking is performed in the two-way mode with the 64-m antenna at Bear Lakes and the 70$\mathrm{m}$ antenna at Ussuriysk. Each tracking session is usually several-hour long, and the observations are separated by 1-3 days. This time interval is much longer than the typical separation between successive desaturations of the reaction wheels (several hours), which makes it difficult to achieve the required accuracy of orbit determination using this type of data alone.

There is another highly valuable type of radio tracking data, which can be obtained when the SRT is performing a scientific observation. The RadioAstron spacecraft does not store any of the radio astronomy data collected by the SRT and transmits it in real time to Earth using its 1.5-m high-gain antenna during every experiment.
The downlink carrier can be synchronized either to the highly stable signal of the on-board H-maser or the uplinked signal of a ground H-maser. The RadioAstron mission is served by two tracking stations capable of receiving the scientific data from the satellite and transmitting an uplink reference signal to it: one at Pushchino (Moscow Region, Russia) and another at Green Bank (West Virginia, USA). Additionally, these tracking stations perform Doppler measurements of the downlink signal during each scientific observation conducted by the satellite. The quality of these measurements is usually much higher than that of the standard radio tracking at the C-band (see Table 3 below). Since the scientific observations are usually performed several times a day and last for about an hour, these measurements provide very valuable data for OD.

A comparison of a posteriori estimated accuracy of radio tracking observations is shown in Table 3. One-way Doppler data provided by the Pushchino and Green Bank stations are much more accurate than the two-way Doppler data obtained by the regular tracking stations of Bear Lakes and Ussuriysk. Relatively large two-way Doppler noise at Bear Lakes and Ussuriysk is explained by the performance of the C-band telemetry, tracking and control system used in the project. However, these stations are also capable of performing range measurements and their two-way Doppler data are free from the contribution of the a priori unknown frequency offset of the on-board Hmaser.

Radio tracking of RadioAstron is supplemented by optical tracking. Routine optical angular observations are conducted by a number of observatories, including the ones of the ISON collaboration, Roscosmos facilities, the MASTER network and many others. More than 40 different telescopes have observed the spacecraft so far and provided optical measurements of right ascension and declination of the spacecraft.

Laser ranging to RadioAstron is also possible and provides distance measurements with cm-level accuracy. However, such observations are performed only occasionally for two reasons. First, the retroreflector array is mounted on the spacecraft in such a way that obtaining laser echoes from it is possible only when the spacecraft is in a specific orientation (or oriented no more than approximately 10 degrees away from it). Because of this, it is almost impossible to perform laser ranging simultaneously with scientific observations, with the exception of the gravity experiment. Second, the spacecraft spends most of the time at distances exceeding $100,000 \mathrm{~km}$, which makes it reachable only to the most powerful laser ranging stations, usually those capable of ranging to the Moon. So far successful laser ranging observations have been performed by the following observatories: Grasse (France), Kavkaz (Russia), Yarragadee, Mt. Stromlo (both in Australia) and Wettzell (Germany).

A test of applying the Planetary Radio Interferometry and Doppler Experiment (PRIDE), which is an OD technique based on observing spacecrafts using VLBI, was 


\begin{tabular}{|c|c|}
\hline Perturbation & Model \\
\hline \multicolumn{2}{|l|}{ Gravity } \\
\hline Static & EGM-96 up to 75 th degree/order \\
\hline Third bodies & JPL DE-405 Sun, Moon and planets \\
\hline Earth tides & IERS-2003 conv. \\
\hline General relativity & IERS-2010 conv. \\
\hline \multicolumn{2}{|l|}{ Surface forces } \\
\hline Solar radiation & 3 parameters $\alpha_{1}, \mu_{1}$ and $\alpha_{2}$ \\
\hline Earth radiation & Applied (constant albedo coeff.) \\
\hline Atmospheric drag & $\begin{array}{l}\text { GOST R } 25645.166-2004 \text { density model, } \\
\text { cannonball force model with one solve-for parameter }\end{array}$ \\
\hline \multicolumn{2}{|l|}{ Desaturation of reaction wheels } \\
\hline Delta V Direction & Telemetry data from the on-board star sensors and attitude file \\
\hline Delta V magnitude & $\begin{array}{l}\text { Telemetry data for the duration of thruster firing } \\
\text { and a priori thrust model }\end{array}$ \\
\hline
\end{tabular}

Table 3: A posteriori estimates of radio tracking errors based on OD results from September 2016 to April 2017. Doppler observations at Bear Lakes and Ussuriysk are received over 1 second integration interval, at Pushchino and Green Bank - normal points with 1 minute averaging were employed.

\begin{tabular}{|c|c|c|}
\hline Tracking station & $\begin{array}{l}\text { Range bias } \\
\text { (RMS), m }\end{array}$ & $\begin{array}{l}\text { Doppler } \\
\text { noise, } \mathrm{mm} / \mathrm{s}\end{array}$ \\
\hline Bear Lake & 2.9 & 1.21 \\
\hline Ussuriysk, 70-m & 13.4 & 4.78 \\
\hline Pushchino, 22-m & $\mathrm{N} / \mathrm{A}$ & 0.19 \\
\hline Green Bank, 140-ft & $\mathrm{N} / \mathrm{A}$ & 0.04 \\
\hline
\end{tabular}

conducted during the in-orbit checkout of the RadioAstron mission Duev et al. (2015). The technique showed the potential for improving the accuracy of estimating the spacecraft state vector. However, PRIDE operations for RadioAstron would require massive involvement of groundbased radio telescopes capable of observing at the $\mathrm{X}$ - and $\mathrm{Ku}$-bands as well as significant data processing resources. Such efforts were deemed unnecessary since other "traditional" OD techniques proved sufficient.

Several types of data collected on board and available via telemetry proved helpful in modeling RadioAstron's dynamics and determining its orbit. The information on the spacecraft attitude and firings of the attitude control thrusters is essential for modeling the perturbations due to SRP and Delta V's that result from momentum dumping of the reaction wheels. Finally, also available via telemetry are the rotation rates of the reaction wheels, which implicitly contain information on the dynamics of the spacecraft center of mass. This information can be extracted in the following way.

Dynamics of the center of mass and attitude dynamics are related to each other by the action of SRP. The attitude control system of the spacecraft uses reaction wheels instead of more commonly applied control moment gyro- scopes. This allows us to estimate the perturbing torque as follows. Given that the spacecraft attitude with respect to an inertial frame is constant on the time interval between $t_{1}$ and $t_{2}$, the change of the angular momentum can be represented in a body-fixed frame as

$$
\sum_{i=1}^{8} I_{i} \mathbf{a}_{i}\left(\Omega_{i}\left(t_{2}\right)-\Omega_{i}\left(t_{1}\right)\right)=\int_{t_{1}}^{t_{2}} \mathbf{M}(t) d t,
$$

where $I_{i}$ is the moment of inertia of the $i$-th wheel, $\mathbf{a}_{i}$ is its axis of rotation, $\Omega_{i}(t)$ is its angular velocity, and $\mathbf{M}(t)$ is the perturbing torque. SRP is usually the only major source of perturbing torque which is responsible for the change of angular momentum of the reaction wheels. Therefore, Equation (3) allows one to obtain the observed value of the SRP torque in a body-fixed frame as long as the SRP variations on the time interval of $\left(t_{1}, t_{2}\right)$ are negligible

$$
\mathbf{M}_{S R P, o}=\frac{1}{t_{2}-t_{1}} \sum_{i=1}^{8} I_{i} \mathbf{a}_{i}\left(\Omega_{i}\left(t_{2}\right)-\Omega_{i}\left(t_{1}\right)\right) .
$$

The corresponding computed value of the SRP torque is given in Equation (2). The computed value depends on the SRP coefficients introduced in Section 2, thus the reaction wheel rotation rates contain valuable information, which otherwise may only be obtained from the orbital dynamics of RadioAstron.

\section{Orbit determination technique}

According to the dynamic model described above, RadioAstron's orbital motion is determined by the following vector of parameters $\mathbf{Q}_{d}=\left\{\mathbf{X}\left(t_{0}\right), \alpha_{1}, \mu_{1}, \alpha_{2}, \Delta \mathbf{v}_{1}, \ldots, \Delta \mathbf{v}_{n}\right\}$, which are, respectively, the initial state vector of the spacecraft, the three SRP coefficients and the $n$ vectors of velocity changes due to desaturations of the reaction wheels, 
and where $n$ is the number of desaturation events in the orbit determination time interval.

The kinematic parameters that affect only the computed values of observables are the session-wise range biases and the frequency offset of the on-board H-maser, also assumed to be piece-wise constant in a way described in Section 6. The latter affects the one-way Doppler observables obtained by the Pushchino and Green Bank tracking stations. Standard orbit determination interval includes at least two full orbits of the spacecraft and usually does not exceed 30 days.

Orbit determination is performed using the batch least squares estimator. The observations of the torque and a priori Delta $\mathrm{V}$ vectors are assumed to be independent from each other and from the tracking data. Therefore, the functional to be minimized can be represented as

$$
\begin{aligned}
\Phi & =\left(\boldsymbol{\Psi}_{o}-\boldsymbol{\Psi}_{c}\right)^{\top} \mathbf{P}\left(\mathbf{\Psi}_{o}-\mathbf{\Psi}_{c}\right)+ \\
& +\sum_{j=1}^{N}\left(\mathbf{M}_{S R P, o}^{j}-\mathbf{M}_{S R P}^{j}\right)^{\top} \mathbf{P}_{j}^{S R P}\left(\mathbf{M}_{S R P, o}^{j}-\mathbf{M}_{S R P}^{j}\right)+ \\
& +\sum_{i=1}^{m}\left(\Delta \mathbf{v}_{i}^{0}-\Delta \mathbf{v}_{i}\right)^{\top} \mathbf{P}_{i}\left(\Delta \mathbf{v}_{i}^{0}-\Delta \mathbf{v}_{i}\right)
\end{aligned}
$$

The first term in Equation (5) gives the contribution of the tracking data $\boldsymbol{\Psi}_{o}$ with corresponding computed values $\boldsymbol{\Psi}_{c}$ and inverted covariance estimate of $\boldsymbol{\Psi}_{o}$ as weight matrix $\mathbf{P}$.

The second term in Equation (5) contains the sum of squares of residual torques, the observed and computed values of which were described in the previous section (Equations (4) and (2)).

The covariance matrices and their inverses, $\mathbf{P}_{S R P}^{j}$, can be estimated using Equation (4), given the estimation errors of the rotation rates of the reaction wheels and the orientations of their axes are known. In practice, however, the weights have to be adjusted to an accuracy level of about $2 \cdot 10^{-6} \mathrm{~N} \cdot \mathrm{m}$, since the perturbing torque model described above is relatively simple and does not always match the accuracy of observations. Fig. 2 shows the agreement between the modeled torque and the observational data. It is clear that for the $X$ and $Z$ components the degree of agreement, in relative terms, is significantly lower than that for the $Y$ component, but it is the latter that contains the dominant part of the torque due to SRP.

The last term of Equation (5) takes into account the a priori information on Delta V's due to desaturations. For each desaturation event the value of net $\Delta \mathbf{v}_{i}^{0}$ is calculated using information on every thruster firing that occurred during that event, including its duration, the fuel pressure and the a priori thrust model. Each of the weight matrices, $\mathbf{P}_{i}$, in (5) is the inverse of the respective covariance matrix given by

$$
\mathbf{C}=\sigma_{d}^{2}\left(\mathbf{E}-\mathbf{e} \cdot \mathbf{e}^{\top}\right)+\sigma_{m}^{2} \mathbf{e} \cdot \mathbf{e}^{\top} .
$$

Here, $\mathbf{e}$ is the unit vector of $\Delta \mathbf{v}$ which has the same direction as $\mathrm{X}$-axis of the spacecraft, $\mathbf{E}$ is a $3 \times 3$ identity
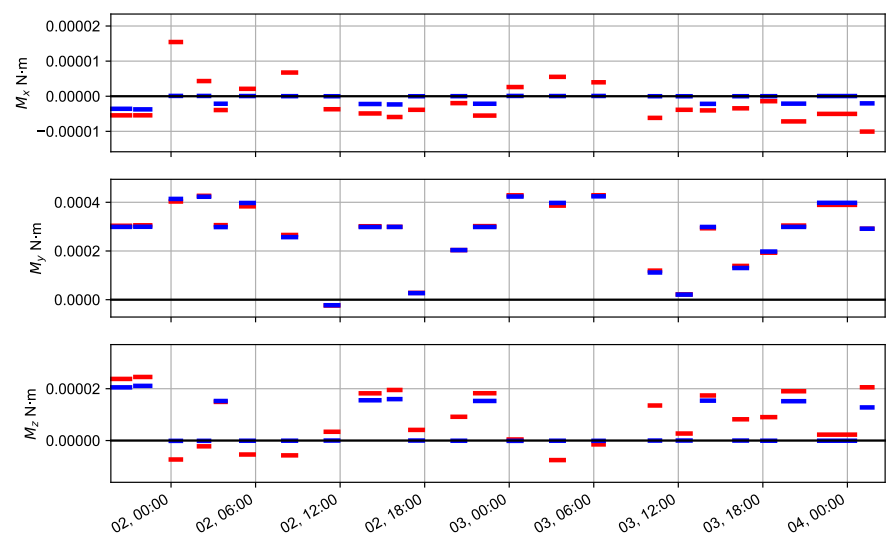

Figure 2: Components of the SRP torque on a $48 \mathrm{hr}$ interval in January 2015: observed (red), computed (blue).

matrix, $\sigma_{m}$ is the error in the estimated magnitude of $\Delta \mathbf{v}$, and $\sigma_{d}$ is the error in the estimate of $\Delta \mathbf{v}$ components in the plane orthogonal to e. The typical value of magnitude error $\sigma_{m}$ used in the OD is $5 \%$ of $\Delta v$, the value of the orthogonal component, $\sigma_{d}$, corresponds to a $0.25 \mathrm{deg}$ error in direction or $4.36 \cdot 10^{-3}$ of $\Delta v$.

In reality the functional is not limited to the one in Equation (5). It accounts also for the a priori information on range biases and the on-board H-maser frequency offset.

\section{ASC correlator overview}

Orbital solutions obtained as a result of the OD procedure outlined above are used mostly for scientific applications, primarily for correlation of VLBI data collected by the mission. In this section we review the operations of one of the correlators capable of processing RadioAstron data, the ASC Correlator. This FX correlator has been developed by the Astro Space Center of the Lebedev Physical Institute (ASC LPI) Likhachev et al. (2017) specifically to support the RadioAstron mission. It is used to process the majority of observations performed by RadioAstron ( $\sim 95 \%)$ and thus provides the largest data set of the so-called residual delays and delay rates, which we will use in the next section to evaluate the orbit determination accuracy.

Correlation of space-VLBI data is performed by the ASC Correlator in two steps, with the second step being necessary due to the orbit determination uncertainties. In order to decrease the residual delay, delay rate and delay acceleration, the first step, or pass, is performed in the socalled "wide" window mode. The window size along the delay is determined by the number of spectral channels, while along the fringe rate it is determined by the integration time. Both parameters are set before the correlation process is started and can be easily adjusted. After a fringe was found, the second correlation pass is performed in a smaller window taking into account the residual delays obtained in the first pass. 
RadioAstron's capability to simultaneously observe at two different wavelengths is of great value for performing the fringe search. In case an observation was performed at two different wavelengths, the residual values obtained as a result of the successful fringe search at the longer wavelength can usually be used to significantly simplify the fringe search at the shorter one.

A delay model for space-VLBI is naturally more complicated than those used for ground-based VLBI. Apart from issues related to the orbit, it has to take into account the fact that the delay rate depends not only on relative velocities of the telescopes but also on the frequency offset of the uplink signal, which is used on board as a reference for the on-board scientific equipment (for the two-way phaselocked loop mode of synchronization), or on the frequency offset of the on-board H-maser (for the one-way mode). The latter, as it turned out, could result in delay rates as high as $30-35 \mathrm{ps} / \mathrm{s}$.

A distinctive feature of the ASC Correlator is its delay model ORBITA2012. This delay model was developed specifically for the ASC Correlator and is capable of calculating the delay up to $O\left(c^{-3}\right)$ terms Vlasov et al. (2012), which provides for using smaller correlation window sizes at the first pass of the correlation process. Moreover, this delay model is capable of taking into account the delay acceleration.

The post-correlation data reduction for all experiments considered in this paper was performed using the PIMA package Petrov et al. (2011). This included baseline fringe fitting, bandpass and amplitude calibration, and averaging the data over time and frequency. The primary goal of the post-correlation processing is to allow for studying the physical properties of the observed objects. The residual values of group delay, delay rate and delay acceleration are a byproduct of this processing. The total delay and its derivatives can be further used for studying the observed objects, e.g. in imaging experiments. This requires accurate calibration of a number of uncertainties, e.g. the rates of the telescope clocks, their trajectories (including that of the SRT), coordinates of the observed sources, propagation media parameters, etc.

The residual delays and their derivatives, which we used in this analysis, were obtained as a result of correlation of the observations of the RadioAstron AGN survey science program and were not used in the OD procedure. The sources observed in this program are compact enough, so that the contribution to residual values due to non-point structure of the sources is negligible.

\section{Orbit determination results}

The orbit determination algorithm outlined in this paper has been implemented and used by the Keldysh Institute of Applied Mathematics (KIAM) to provide RadioAstron users with a posteriori orbits required to correlate VLBI data collected by the mission. The majority of observations performed since 2014 and a significant portion of earlier observations were correlated using orbits produced with this version of the algorithm. At the early stages of the mission, i.e. for experiments conducted before 2014, a previous, less sophisticated version of the OD algorithm was used.

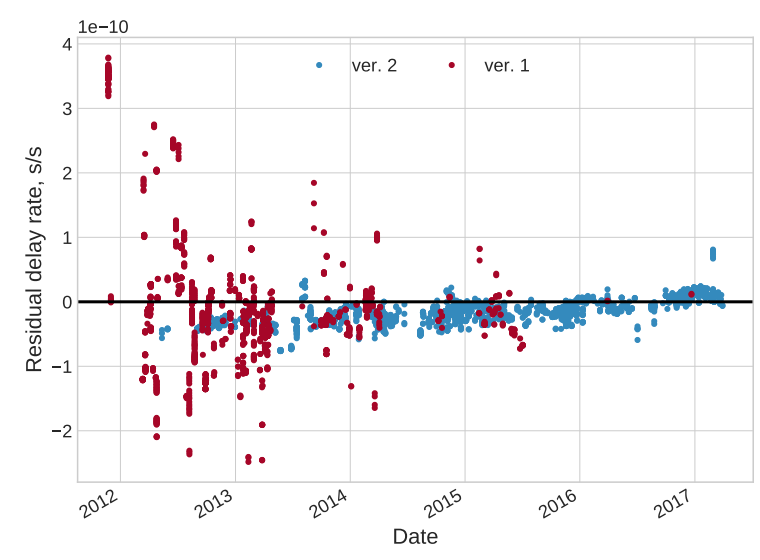

Figure 3: Residual delay rates obtained by the ASC Correlator using two versions of the orbit: generic algorithm, version 1 (red dots); new algorithm, version 2 (blue dots).

A statistically significant correlation detected between signals recorded by the RadioAstron space radio telescope and ground-based radio telescopes yields a set of three residual values, that of delay, delay rate and delay acceleration. In this paper we focus exclusively on the delay rates for the following reasons. First, data collected by the space radio telescope are not time-tagged on board, i.e. synchronization of the on-board H-maser time scale with that of UTC is possible only indirectly, by means of time-tagging the data stream flowing from the satellite by the receiving tracking station. Second, there is a non-zero probability of inconsistent synchronization of ground radio telescopes with UTC. And finally, as shown below, there are a number of significant orbit-unrelated factors, which can contribute to the residual delay rate also affecting the residual delay. All this makes it difficult to separate the contribution to residual delay due to errors in the orbit from those due to the involved clocks. On the other hand, possible contributions to the residual delay rate are much more predictable. Time synchronization by means of a tracking station does not affect the delay rate, since any change in the delay it may introduce remains constant during every "scan" (file) of the recorded data. Drifts of ground-based clocks are usually much smaller than the residual delay rates we observe and, moreover, when an experiment involves more than one ground radio telescope, relative drifts of the ground-based clocks can be estimated by performing fringe searches on ground-only baselines. Finally, the clock drift of the spacecraft clock can be estimated independently in the process of orbit determination. 
Fig. 3 shows the residual delay rates obtained by the ASC Correlator as a result of processing the observations performed by the RadioAstron mission up to early 2017. Each data point in Fig. 3 corresponds to a value of the residual delay rate determined by the correlator as a result of correlating two data scans, i.e. units of data typically of $\sim 10$ min duration, one of which was recorded by the RadioAstron space radio telescope and the other by a ground radio telescope that participated in the experiment. Blue dots denote the residual delay rates obtained with version 2 orbits, i.e. those determined with the algorithm outlined in Section 4. Red dots denote the values obtained with verion 1 orbits, i.e. determined with a generic OD algorithm and a simpler dynamic model of the spacecraft. The earlier version of the algorithm uses a simple cannonball model to estimate the SRP and thus ignores its attitude dependence. Also, the velocity changes due to momentum dumps are not estimated in the generic algorithm but set to their a priori values, $\Delta \mathbf{v}_{i}^{0}$. For each data point of Fig. 3 it is assumed that RadioAstron is the first antenna while the ground radio telescope is the second one, i.e. the depicted residual delay rates are relative to the space-based antenna.

All the observations used to obtain the residual delay rates of Fig. 3 were conducted in the so-called "one-way" mode, which is characterized by the following two conditions: a) the on-board scientific equipment is synchronized to the reference signal of the on-board H-maser; b) the carrier of the downlink signal used to transfer the science data from the spacecraft to a tracking station is also synchronized to the reference $\mathrm{H}$-maser signal.

It is clear from Fig. 3 that the variation of the residual delay rates obtained with version 2 of the OD algorithm is substantially smaller than that of the delay rates obtained with the generic algorithm. The RMS of the residual delay rates are $2.463 \times 10^{-11}$ for version 2 and $1.097 \times 10^{-10}$ for version 1 . It is also clear that the residual delay rates obtained with version 2 orbits are offset from zero, with the mean value equal to $1.663 \times 10^{-11}$ and also reveal a long-term linear trend (Fig. 4). The latter is clearly an indication of a systematic effect which cannot be attributed to an OD error since these residual values were obtained using a large number of independent orbit solutions and an even greater number of observations of radio sources scattered all over the sky and observed at various projected baselines.

Let us consider the impact of the orbit errors on the calculated value of the group delay reported by the correlator. Denote the spacecraft state vector in a geocentric inertial reference frame according to the orbital solution by $\mathbf{X}(t)=\left(\mathbf{r}_{1}(t)^{\top}, \mathbf{v}_{1}(t)^{\top}\right)^{\top}$, the true state vector by $\overline{\mathbf{X}}(t)=\left(\overline{\mathbf{r}}_{1}^{\top}(t), \overline{\mathbf{v}}_{1}^{\top}(t)\right)^{\top}$ and the difference between the two by $\delta \mathbf{X}(t)=\overline{\mathbf{X}}(t)-\mathbf{X}(t)=\left(\delta \mathbf{r}_{1}(t)^{\top}, \delta \mathbf{v}_{1}(t)^{\top}\right)^{\top}$. Provided that the requirements to OD accuracy stated in Section 1 are met, the error in the Geocentric Coordinate Time (TCG) delay rate Vlasov et al. (2012) due to OD errors can be given by the following equation, which is

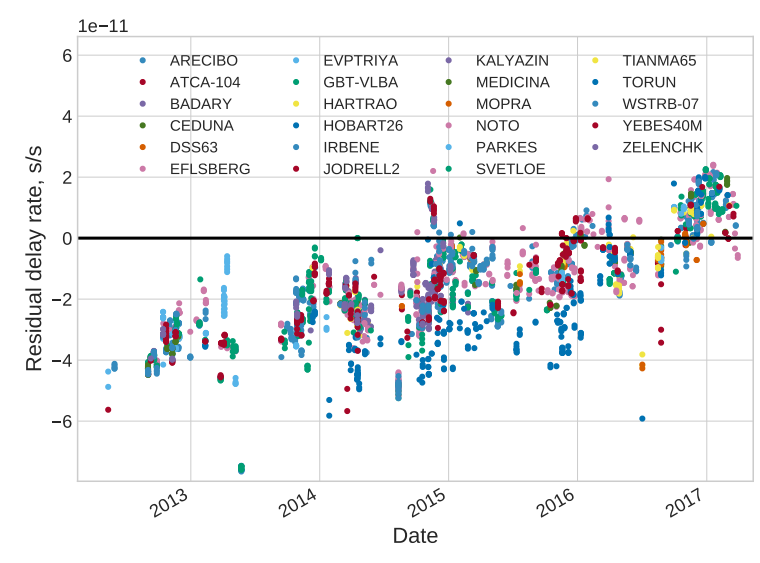

Figure 4: The residual delay rates obtained using version 2 orbits. Color indicates the ground radio telescope of the baseline.

accurate to at least $10^{-14}$ :

$$
\frac{d}{d t} \delta \tau_{0}=-\frac{1}{c} \mathbf{k} \cdot \delta \mathbf{v}_{1}\left(t_{2}\right)
$$

where $\mathbf{k}$ is a unit vector pointing in the direction of the source (the aberration factor can be omitted provided our accuracy requirements).

The delay understood by the correlator is a difference of proper time intervals counted by the station clocks from the moment of synchronization to the moment of signal reception. Thus the estimate for the TCG delay rate given by Equation (6) may be insufficiently accurate, especially in the case when one of the stations is orbiting the Earth. Assuming the clocks were synchronized at $t_{s}$ the delay should be as follows

$$
\begin{array}{r}
\tau=\tau_{0}+\frac{1}{c^{2}} \int_{t_{s}}^{t_{2}}\left(\frac{v_{2}^{2}}{2}+U\left(\mathbf{r}_{2}\right)\right) d t-\frac{1}{c^{2}} \int_{t_{s}}^{t_{1}}\left(\frac{v_{1}^{2}}{2}+U\left(\mathbf{r}_{1}\right)\right. \\
\left.+V\left(\mathbf{R}_{1}\right)-V\left(\mathbf{R}_{\oplus}\right)-\frac{\partial V\left(\mathbf{R}_{\oplus}\right)}{\partial \mathbf{R}} \mathbf{r}_{1}\right) d t
\end{array}
$$

where $\tau_{0}$ is the TCG delay, $t_{1}$ and $t_{2}$ are, respectively, the moments of signal reception by station 1 and station 2 , $U(\mathbf{r})$ is the Newtonian potential of the Earth at position $\mathbf{r}, V$ is the sum of the Newtonian potentials of all bodies of the Solar System excluding the Earth, $\mathbf{R}_{1}$ is the barycentric position of the spacecraft, and $\mathbf{R}_{\oplus}$ is the barycentric position of the geocenter. The tidal potential is neglected for the ground-based station (i.e. station 2), following the recommendations of Petit and Luzum (2010).

The change of the delay given by Equation (7) due to errors in the spacecraft position, $\delta \mathbf{r}_{1}$, and velocity, $\delta \mathbf{v}_{1}$, can be represented as

$$
\begin{aligned}
\delta \tau\left(\delta \mathbf{r}_{1}, \delta \mathbf{v}_{1}\right)= & \left.\delta \tau_{0}\left(\delta \mathbf{r}_{1}, \delta \mathbf{v}_{1}\right)\right) \\
& -\frac{1}{c^{2}} \int_{t_{s}}^{t_{1}}\left(\mathbf{v}_{1} \cdot \delta \mathbf{v}_{1}-U \frac{\mathbf{r}_{1} \cdot \delta \mathbf{r}_{1}}{r_{1}^{2}}\right) d t
\end{aligned}
$$


where the tidal term, which can be approximated by $\frac{1}{2} \sum_{i=1}^{3} \sum_{\text {: }}^{\text {: }}$ has been neglected since for RadioAstron's orbit and the expected magnitude of OD errors, the contribution of this term to the delay rate is less than $10^{-19}$. Therefore the correction to the delay rate can be written as

$$
\frac{d}{d t} \delta \tau=\frac{d}{d t} \delta \tau_{0}-\frac{\mathbf{v}_{1} \cdot \delta \mathbf{v}_{1}}{c^{2}}-U \frac{\mathbf{r}_{1} \cdot \delta \mathbf{r}_{1}}{c^{2} r_{1}^{2}}
$$

Provided that OD errors do not exceed the values stated in Section 1, the last two terms on the right-hand side of Equation (9) contribute at most $10^{-13}$. Therefore, if we aim at evaluating the correction to the delay rate with an accuracy of $10^{-13}$, these two terms can be neglected. Adding to this the correction due to the on-board H-maser clock drift, $h_{1}(t)$ and that of the ground-based station, $h_{2}(t)$, we arrive at the following equation

$\frac{d}{d t} \delta\left(\left(t_{2}-t_{s}\right)_{p}-\left(t_{1}-t_{s}\right)_{p}\right)=\frac{1}{c} \mathbf{k} \cdot \delta \mathbf{v}_{1}\left(t_{2}\right)-h_{1}\left(t_{1}\right)+h_{2}\left(t_{2}\right)$

which can be used to investigate the spacecraft velocity error, provided it is larger than $\sim 10^{-13}$ but within the limit given in Section 1, i.e. less than $\sim 10^{-10}$. The two additional assumptions needed to justify the use of Equation (10) for this purpose are: a) the position and velocity of the ground-based station (\#2) are known much better than those of the spacecraft and b) the error of modeling the contribution of the propagation media to the delay rate is less than $10^{-13}$. While the first assumption is undoubtedly true, the second may be violated in rare cases of extreme weather conditions.

The delay model implemented by the ASC Correlator assumes that the spacecraft and station clocks are ideal, i.e. $h_{1}(t) \equiv 0$ and $h_{2}(t) \equiv 0$. Therefore, any instrumental effect affecting the clock drift, if present, would manifest itself in an extra residual delay rate reported by the correlator.

If we assume that no other systematic effects are present, each data point shown in Fig. 3 corresponds to the righthand side of Equation (10) evaluated at a certain time using a certain orbital solution plus an error of the delay rate determination inherent to the fringe search procedure. Therefore, if we are able to evaluate the clock drifts of the space and ground-based station clocks, and apply the corresponding corrections to each data point of Fig. 3, the result will be the spacecraft velocity errors projected onto the directions to the observed celestial sources.

We assume that the ground-based station clock drift, $h_{2}(t)$, is always well below the level of $10^{-13}$ and thus can be neglected, which is justified by the results of fringe searches at ground-only baselines. Under this assumption, any clock drift of the ground station, if present, will be attributed to the error in the spacecraft velocity. The drift of the on-board clock, $h_{1}(t)$, is estimated as part of the OD process in the way outlined below.

All the observations used for the present analysis were performed, as noted above, in the so-called one-way mode,

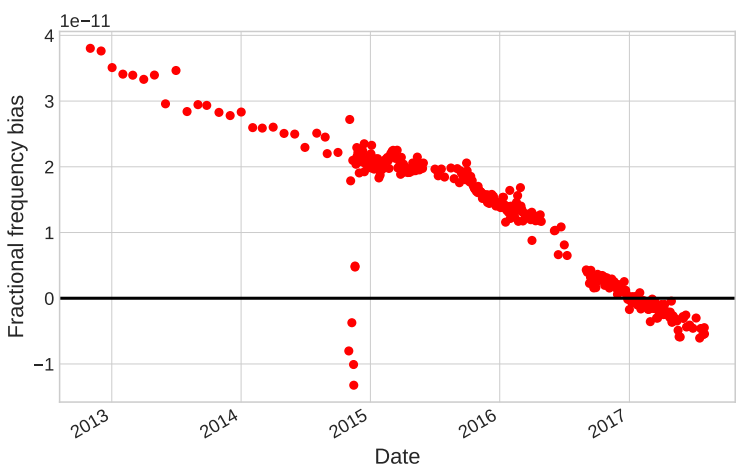

Figure 5: Evolution of the RadioAstron on-board H-maser fractional frequency bias. Each value is obtained as part of an orbital solution. The density of points before and after October 2014 is different because of a change of the frequency bias estimation strategy.

i.e. when both the on-board science equipment and the downlink carrier are locked to the on-board H-maser reference signal. Therefore, if the on-board H-maser output frequency is biased, i.e. there is a non-zero clock drift, both the science data, which eventually results in the residual delay rates used in our analysis, and the downlink carrier frequency measurements, which are used in OD, will be affected by this same frequency bias.

In order to properly fit one-way Doppler measurements in the OD process, frequency bias of the tracking station $\mathrm{H}$-maser and that of the on-board $\mathrm{H}$-maser have to be taken into account. For each of the two tracking stations we used a priori values of the frequency biases, which we derived from long-term series of measured differences between the station's local time, synchronized to its H-maser, and the GPS time. We assume the on-board H-maser fractional frequency bias, $\Delta f / f$, to be identical to that of the downlink carrier, and estimate the latter in the OD process.

The on-board H-maser frequency biases obtained this way are shown in Fig. 5. The bias evolution is almost linear over more than 4 years of the analyzed data. In late October 2014 an accident occurred on board after which the onboard H-maser started experiencing a power surge every time one of the telemetry and command (T\&C) transponder was turned on. Before that accident our approach to estimating the on-board H-maser frequency bias was to treat it as constant over the whole OD interval. After the accident we changed our strategy and estimated the bias independently for each interval between the T\&C link transponder on/off switches, which usually coincided with radio tracking from Bear Lakes or Ussuriysk stations. This change of strategy explains the different density of data points in Fig. 5 before and after October 2014.

Using the above assumption that the downlink carrier fractional frequency bias, $\Delta f / f$, is equal to that of the on-board H-maser, i.e to the on-board clock drift, $h_{1}$, we can substract it from the residual delay rates using Equa- 
tion (10). The residual delay rates obtained by applying this correction are shown in Fig. 6 (cf. Fig. 4). With the correction applied the RMS of the residual delay rates reduces to $1.239 \times 10^{-11}$ and its mean value reduces from $1.663 \times 10^{-11}$ to $3.735 \times 10^{-12}$.

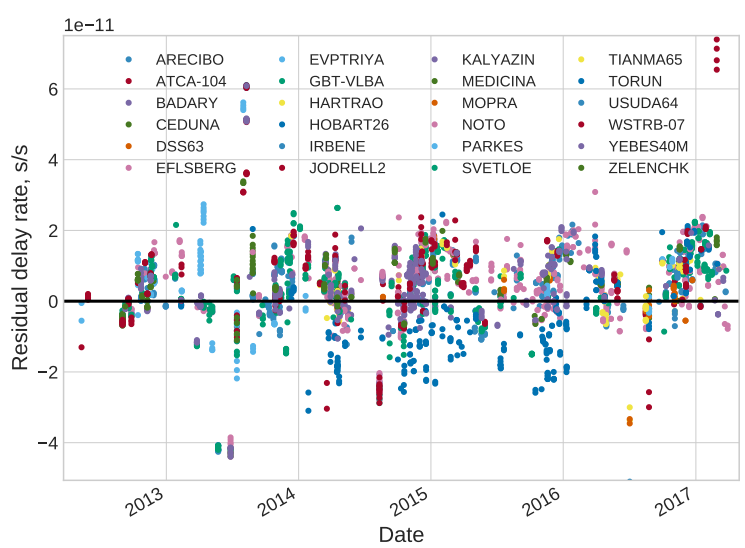

Figure 6: The residual delay rates obtained using version 2 orbits and corrected for the on-board clock drift. Color indicates the ground radio telescope of the baseline.

According to Equation (10), we expect each value of the residual delay rate corrected for the spacecraft clock drift to be equal to the corresponding spacecraft velocity error projected onto the direction of the observed radio source. Therefore, we expect them to be largely uncorrelated and symmetrically distributed with respect to zero. However, the values shown in Fig. 6 still reveal significant systematic effects, i.e. the average value is $1.12 \mathrm{~mm} / \mathrm{s}$ in terms of velocity and there are noticeable variations with a period of about 1 year. The histogram of the data is shown on the left-hand side of Fig. 8.

A more detailed analysis of the data shown in Fig. 6 using the Lomb-Scargle periodogram method reveals that, in addition to the annual variation, the residual delay rates are modulated with a period of about 8.8 day (Fig. 7), which is close to the average orbital period.

The periodic variations visible in Fig. 6 and confirmed on the left panel of Fig. 7 are present in the original data and not due to the applied clock correction $h_{1}(t)$.

In an attempt to understand the origin of these periodicities we tried several hypotheses. We were able to determine that the periodic patterns in the residual delay rates can be noticeably reduced by applying the following correction

$$
\delta \dot{\tau}=\frac{1}{c^{2}}\left(U_{\odot}(\mathbf{R})-U_{\odot}\left(\mathbf{R}_{\oplus}\right)\right)
$$

Here, $U_{\odot}$ is the Newtonian potential of the Sun, $\mathbf{R}$ is the barycentric position of the RadioAstron spacecraft during the experiment and $\mathbf{R}_{\oplus}$ is the barycentric position of the geocenter.
We are working on developing a full understanding of why the correction of Equation (11) is required. However, its origin is likely due to either an inconsistency between the models of orbital motion or the delay model used by the correlator, or both. This can be concluded from the following reasoning. Orbital solutions are provided in the geocentric coordinate system and the Terrestrial Time (TT) scale, as the correlator delay model expects. In that case the effect of the solar gravitational potential on the delay is described by the last three terms in the second integral on the right-hand side of Equation (7), with the potential $V$ replaced by $U_{\odot}$. For the delay rate it differs from the correction in Equation (11) by the third term, $\partial U_{\odot}\left(\mathbf{R}_{\oplus}\right) / \partial \mathbf{R} \cdot \mathbf{r}_{1} / c^{2}$, which is approximately equal to the expression in Equation (11) and makes the tidal effect of the solar gravitational potential almost negligible in the case of RadioAstron. Therefore, the correction in the form of Equation (11) cannot be due to an unaccounted effect of the solar gravitational potential but can only arise due to an inconsistency in the computation of delays using the provided orbit solutions.

The residual delay rates after application of the correction of Equation (11) are shown in Fig. 9. In addition to overall reduction of delay rate variation, it is now clear that part of the data points, i.e. those that correspond to RadioAstron-Torun baselines, have an appreciable bias of about $-1.9 \times 10^{-11}$, which can reasonably be attributed to the clock drift of this ground radio telescope.

Excluding the Torun data, which constitute $8.2 \%$ of the dataset, we arrive at the mean value of the residual delay rates of just $4.387 \times 10^{-13}$ and its RMS of $9.573 \times 10^{-12}$. The distribution of the data also becomes more symmetric (Fig. 8).

In order to investigate the statistical properties of the residual delay rates shown in Fig. 9 further, we exclude the data of experiments conducted in the summer periods, i.e. between the 1st of June and the 1st of September. These experiments constitute $10.4 \%$ of data, but their RMS, equal to $2.307 \times 10^{-11}$, is more than 5 times larger than that of the rest of the data. Degrade orbit determination accuracy is definitely one of the main reasons why the summer experiments exhibit larger residual delay rates. The number of scientific observations drops significantly during summer, mainly due to constrains on the spacecraft attitude with respect to the Sun, which reduce the visibility of radio sources of interest. The lack of experiments reduces the amount of Doppler data from the tracking stations which, in turn, affects the orbit accuracy.

After excluding the summer experiments and filtering out outliers, which, for the $5 \sigma$ threshold, constitute $1.15 \%$ of the original data, we obtain a dataset which contains $88.4 \%$ of the initial number of residual delay rates. For this subset of the data the mean residual delay rate is $4.428 \times$ $10^{-13}$ and the RMS is $4.255 \times 10^{-12}$. This corresponds to the standard deviation of the projected spacecraft velocity error, $\mathbf{k} \cdot \delta \mathbf{v}_{1}$, of $1.275 \mathrm{~mm} / \mathrm{s}$.

To summarize, we obtained a dataset of the spacecraft 

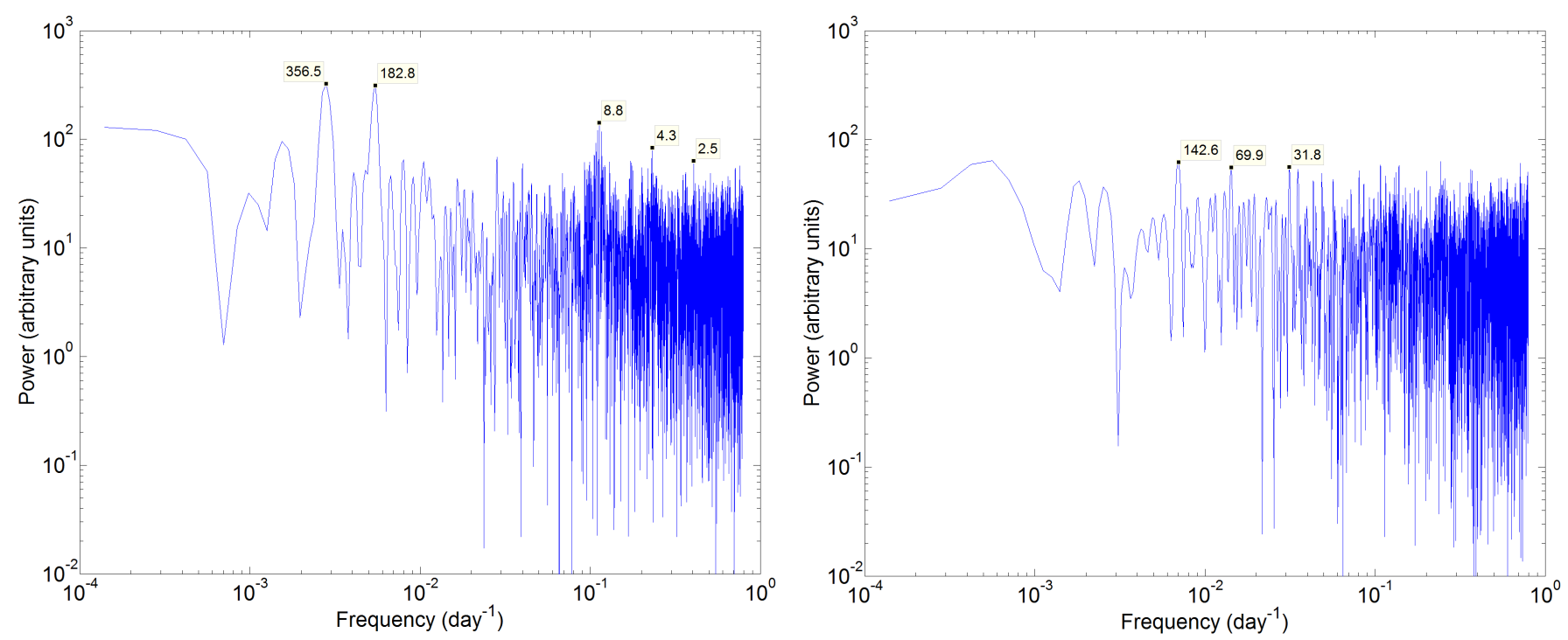

Figure 7: A Lomb-Scargle periodogram of residual delay rates corrected for the on-board H-maser drift before (left) and after (right) application of the correction of Equation (11). Significant spikes are annotated with the periods (in days) corresponding to their frequencies.
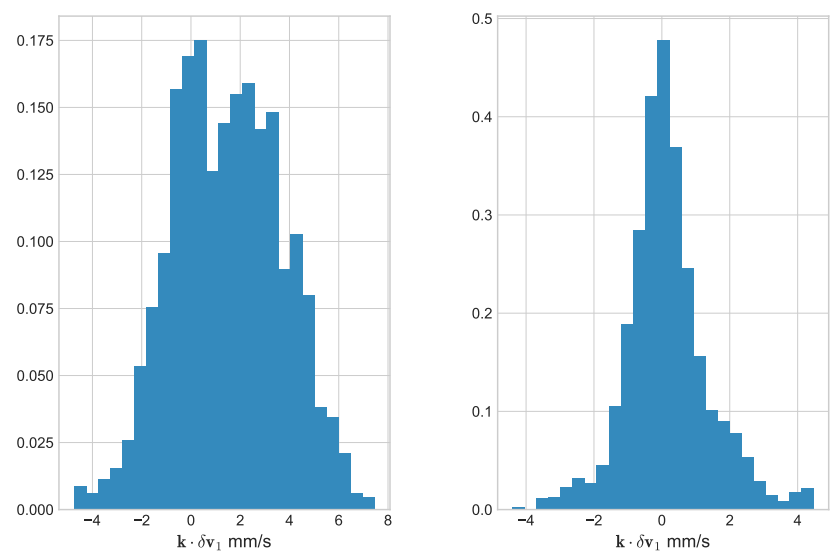

Figure 8: Histograms of the residual delay rates after application of the correction for the on-board clock drift. Left: the additional correction of Equation (11) is not applied. Right: the correction of Equation (11) is applied. Experiments with the Torun ground radio telescope are excluded.

velocity error estimates projected onto the directions of the observed sources, $\mathbf{k} \cdot \delta \mathbf{v}_{1}$. We assumed that clock drifts of ground-based VLBI stations that provided the ground legs of the baselines are negligible, the on-board clock drift is equal to the estimated value of the downlink carrier fractional frequency offset, and we applied the correction of Equation (11) to remove systematic patterns in the residual delay rates due to suspected inconsistencies between the orbit and correlator delay models. Now, in order to obtain information on the spacecraft velocity error itself, $\delta \mathbf{v}_{1}$, and not only on its projection, $\mathbf{k} \cdot \delta \mathbf{v}_{1}$, we represent the residual delay rates as a function of the angle, $\alpha$, between the direction to the observed source, $\mathbf{k}$, and the direction

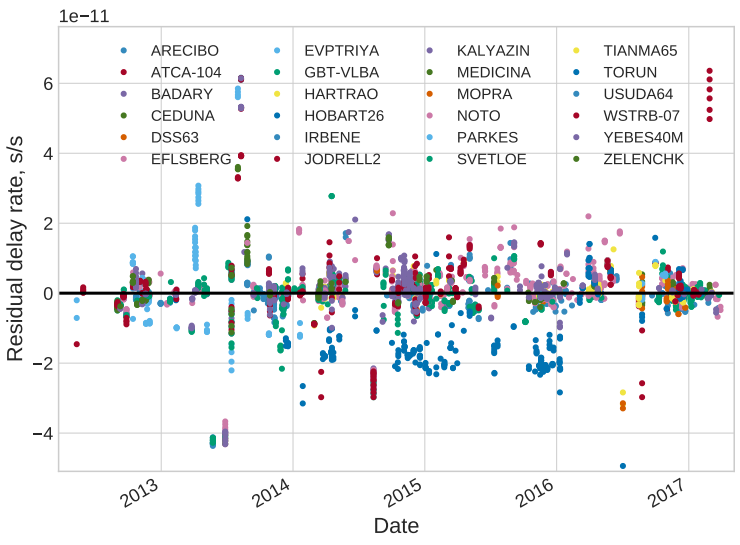

Figure 9: The residual delay rates obtained using version 2 orbits corrected for the on-board clock drift and for the correction of Equation (11). Color indicates the ground radio telescope of the baseline.

to the tracking station that was receiving data from the spacecraft.

Since every science observation is accompanied by simultaneous Doppler measurements performed by the tracking station used to receive the science data, the largest errors are to be expected in the velocity components that belong to the plane which is normal to the tracking station direction. However, contrary to this expectation, Fig. 10 does not reveal any significant dependence of the projected velocity error, $\mathbf{k} \cdot \delta \mathbf{v}_{1}$, on $\alpha$. A possible reason for this is that the corrected residual delay rate, and thus $\mathbf{k} \cdot \delta \mathbf{v}_{1}$, contains errors due to inaccurate estimation of the on-board clock drift, $h_{1}(t)$, and the assumption on the ground station clocks that $h_{2}(t) \equiv 0$, both of which do not depend 
on the source direction, $\mathbf{k}$, and may provide significant contributions to the residual delay rates.

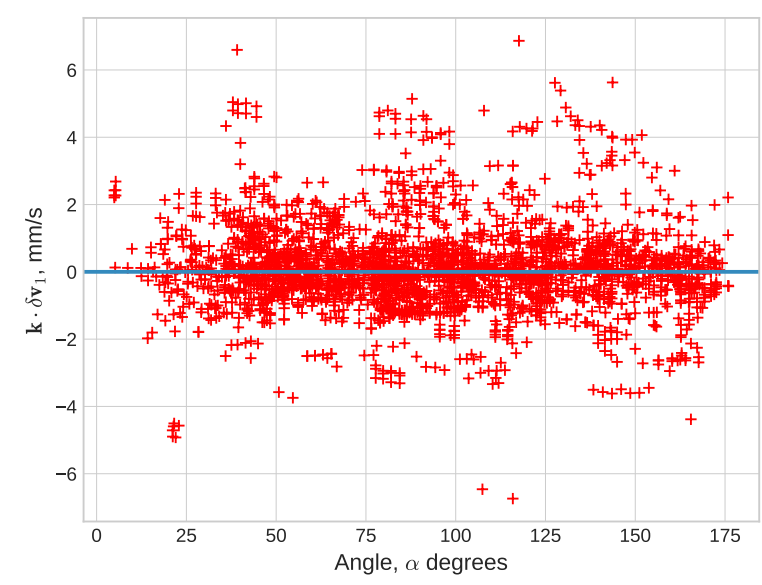

Figure 10: Dependence of the residual delay rates on the angle between the direction to observed source and the direction from the spacecraft to the tracking station which was used to receive the data of that observation

We can estimate the velocity error along the "worst" directions using the dependence of the projected velocity error, $\mathbf{k} \cdot \delta \mathbf{v}_{1}$, on $\alpha$. For the experiments, in which $75^{\circ} \leq \alpha \leq 105^{\circ}$, the $\mathrm{RMS}$ of $\mathbf{k} \cdot \delta \mathbf{v}_{1}$ is equal to 1.359 $\mathrm{mm} / \mathrm{s}$. Assuming the spacecraft velocity is determined not worse along other directions the error ellipsoid of $\delta \mathbf{v}_{1}$ is confined by the one corresponding to the covariance matrix $\operatorname{diag}\left(1.85 \mathrm{~mm}^{2} / \mathrm{s}^{2}, 1.85 \mathrm{~mm}^{2} / \mathrm{s}^{2}, 1.85 \mathrm{~mm}^{2} / \mathrm{s}^{2}\right)$.

In order to obtain an independent estimate of the velocity error, we compared 55 independent adjacent orbit solutions from 2014 to 2018 for discontinuity in velocity. If the first solution was obtained for, e.g., the time interval of $\left(t_{1}, t_{2}\right)$ and the second one for $\left(t_{2}, t_{3}\right)$, the spacecraft state vector is compared at $t_{2}$. The time of comparison, $t_{2}$, is thus either the beginning or the end of an OD interval. Unlike the case of the residual delay rates, these times do not correspond to any tracking activity and, therefore, the state vectors evaluated at these moments contain additional prediction errors.

The 3D RMS of the 54 velocity differences computed this way is $4.38 \mathrm{~mm} / \mathrm{s}$. Just like the residual delay rates, these velocity differences exhibit significant increase in magnitude in the summer months due to the decrease of the amount of tracking data. If we exclude the summer data, we arrive at a dataset of 42 points and 3D RMS of the velocity differences of $3.52 \mathrm{~mm} / \mathrm{s}$.

However, we should take into account that such differences contain errors of two independent orbit solutions, therefore, their variance is as sum of variances of two orbit solutions. Thus, we can conclude that the $3 \mathrm{D}$ RMS of the velocity error of a single solution is $3.10 \mathrm{~mm} / \mathrm{s}$ for the full dataset and $2.53 \mathrm{~mm} / \mathrm{s}$ for its summer-free subset.

The RMS values of the spacecraft velocity error pro- vided by the discontinuity analysis and those obtained using the residual delay rates are similar. However, since the former contain, as noted above, additional prediction errors, we expected them to be higher. This may be an indication of the fact that even after the corrections to the residual delay rates have been applied, clock-related errors still give a significant contribution to these data, resulting in overestimated values of velocity errors.

\section{Discussion}

We obtained two independent estimates of the velocity errors, the one using the comparison of adjacent independent orbits and the other via the residual delay rate analysis. The obtained results agree very well. However, in order to obtain the velocity error estimates using the residual delay rate data, we had to apply two non-trivial corrections to the data.

The first correction is to take into account an instrumental effect of the spacecraft clock drift. The significance of the on-board H-maser clock drift is evident from the combined analysis of two-way range and Doppler tracking data together with the one-way Doppler measurements of the downlink carrier synchronized to the on-board $\mathrm{H}-$ maser. Since the ASC Correlator assumes the clocks to be ideal, i.e. any non-zero clock drift goes directly into the residual delay rate, the necessity to correct for the on-board clock drift is well justified. This correction, however, introduces additional errors into the residual delay rate data due to the way it is estimated in the process of OD.

The necessity of the second correction, that of Equation (11), is also clear as it significantly improves the residual delay rates by cleaning them from artificial periodic patterns. However, its nature is currently not as well understood. We suspect the necessity of this correction is due to an inconsistency between the models of orbital motion or the delay model used by the correlator, or both. Investigation of the physical cause behind this corrections is beyond the scope of the present work and would require a deeper insight in the experimental data accumulated by the mission.

The residual delay rate data provide a unique means of estimating velocity errors of a space-VLBI spacecraft. If the mission performs observations of radio sources distributed all over the sky, the method effectively provides estimates of all components of the spacecraft velocity error, as shown in Fig. 10.

The OD accuracy evaluation of the only previous spaceVLBI mission of VSOP/HALCA, given by You et al. (1998), did not include an analysis of the residual delay rate data. However, the overall OD accuracy of the HALCA spacecraft, obtained with traditional methods, was better than that of RadioAstron. This can be easily understood as the HALCA spacecraft was tracked by a larger number of tracking stations, each possibly with better instrumental stability than those involved in RadioAstron. 


\section{Conclusion}

We outlined our approach to orbit determination of the RadioAstron spacecraft. The method includes an inhouse developed SRP model and an algorithm to take into account the accumulated angular momentum of reaction wheels to improve our knowledge of the dynamics of the spacecraft center of mass. We tested the performance of this OD method using the unique "tracking data" available only for a space-VLBI spacecraft, i.e. the residual delay rates, which in our case were obtained by the ASC Correlator of the RadioAstron mission as a result of processing the data of VLBI observations of celestial radio sources performed by the RadioAstron spacecraft together with ground radio telescopes. This analysis allowed us to conclude that the OD method we developed provides up to 11 times more accurate orbital solutions in terms of velocity and residual delay rates as compared to a generic OD algorithm.

Using the residual delay rate data we have obtained an estimate of the standard deviation for every component of the spacecraft velocity error. The residual delay rates obtained in non-summer observations, which constitute $88.4 \%$ of all data, exhibit a standard deviation of the spacecraft velocity error of $1.4 \mathrm{~mm} / \mathrm{s}$ or less for each velocity component. This result is consistent with an independent a posteriori estimate of the spacecraft velocity error obtained using the analysis of velocity differences computed at the boundaries of adjacent orbital solutions.

\section{Acknowledgements}

The RadioAstron project is led by the Astro Space Center of the Lebedev Physical Institute of the Russian Academy of Sciences and the Lavochkin Scientific and Production Association under a contract with the Russian Federal Space Agency, in collaboration with partner organizations in Russia and other countries including Keldysh Institute of Applied Mathematics of the Russian Academy of Sciences. Partly based on observations performed with radio telescopes of IAA RAS (Federal State Budget Scientific Organization Institue of Applied Astronomy of Russian Academy of Sciences). Partly based on the Evpatoria RT-70 radio telescope (Ukraine) observations carried out by the Institute of Radio Astronomy of the National Academy of Sciences of Ukraine under a contract with the State Space Agency of Ukraine and by the National Space Facilities Control and Test Center with technical support by Astro Space Center of Lebedev Physical Institute, Russian Academy of Sciences. Partly based on observations with the 100-m telescope of the MPIfR (MaxPlanck-Institute for Radio Astronomy) at Effelsberg. Partly based on observations with the Medicina (Noto) telescope operated by INAF - Istituto di Radioastronomia. The National Radio Astronomy Observatory is a facility of the National Science Foundation operated under cooperative agreement by Associated Universities, Inc. The Green
Bank Observatory is a facility of the National Science Foundation operated under cooperative agreement by Associated Universities, Inc. The Arecibo Observatory is operated by SRI International under a cooperative agreement with the National Science Foundation (AST-1100968), and in alliance with Ana G. Mendez-Universidad Metropolitana, and the Universities Space Research Association. The Australia Telescope Compact Array (Parkes radio telescope / Mopra radio telescope / Long Baseline Array) is part of the Australia Telescope National Facility which is funded by the Commonwealth of Australia for operation as a National Facility managed by CSIRO. This work is based in part on observations carried out using the 32 -meter radio telescope operated by Torun Centre for Astronomy of Nicolaus Copernicus University in Torun (Poland) and supported by the Polish Ministry of Science and Higher Education SpUB grant. Results of optical positioning measurements of the Spektr-R spacecraft by the global MASTER Robotic Net Lipunov et al. (2010), ISON collaboration, and Kourovka observatory were used for spacecraft orbit determination in addition to mission facilities.

\section{References}

Duev, D.A., Zakhvatkin, M.V., Stepanyants, V.A., et al., 2015. RadioAstron as a target and as an instrument: Enhancing the Space VLBI mission's scientific output. Astronomy \& Astrophysics 573, A99. doi:10.1051/0004-6361/201424940, arXiv:1411.4576.

Kardashev, N.S., Khartov, V.V., Abramov, V.V., et al., 2013. "RadioAstron"-A telescope with a size of $300000 \mathrm{~km}$ : Main parameters and first observational results. Astronomy Reports 57, 153-194. doi:10.1134/S1063772913030025.

Likhachev, S.F., Kostenko, V.I., Girin, I.A., et al., 2017. Software Correlator for Radioastron Mission. Journal of Astronomical Instrumentation 6, 17. doi:10.1142/S2251171717500040, arXiv: 1706.06320.

Lipunov, V., Kornilov, V., Gorbovskoy, E., et al., 2010. Master Robotic Net. Advances in Astronomy 2010, 349171. doi:10.1155/ 2010/349171, arXiv:0907.0827.

Litvinov, D., Rudenko, V., Alakoz, A., et al., 2018. Probing the gravitational redshift with an Earth-orbiting satellite. Physics Letters A 382, 2192 - 2198. doi:10.1016/j.physleta.2017.09. 014. Special Issue in memory of Professor V.B. Braginsky.

Nunes, N., Bartel, N., Bietenholz, M., et al., 2019. The gravitational redshift monitored with RadioAstron from near Earth up to $350,000 \mathrm{~km}$. Advances in Space Research doi:10.1016/j.asr. 2019.03.012, arXiv:1904.01060.

Petit, G., Luzum, B., 2010. IERS Conventions (2010). Technical Report. Verlag des Bundesamts fr Kartographie und Geodsie. IERS Technical Note ; 36

Petrov, L., Kovalev, Y.Y., Fomalont, E.B., et al., 2011. The Very Long Baseline Array Galactic Plane Survey - VGaPS. The Astronomical Journal 142, 35. doi:10.1088/0004-6256/142/2/35, arXiv: 1101.1460 .

Vlasov, I.Y., Zharov, V.E., Sazhin, M.V., 2012. Signal delay in the RadioAstron ground-space interferometer. Astronomy Reports 56, 984-987. doi:10.1134/S1063772912120086.

You, T.H., Ellis, J., Mottinger, N., 1998. Navigation of the space VLBI mission-HALCA, in: Stengle, T. (Ed.), AAS/GSFC 13th International Symposium on Space Flight Dynamics, pp. 841-856.

Zakhvatkin, M.V., Ponomarev, Y.N., Stepanyants, V.A., et al., 2014. Navigation support for the RadioAstron mission. Cosmic Research 52, 342-352. doi:10.1134/S0010952514050128. 